\title{
Publisher Correction: Metabolic engineering of Escherichia coli for shikimate pathway derivative production from glucose-xylose co-substrate
}

\author{
Ryosuke Fujiwara (1), Shuhei Noda, Tsutomu Tanaka (1) \& Akihiko Kondo
}

Correction to: Nature Communications https://doi.org/10.1038/s41467-019-14024-1, published online 14 January 2020.

In the original version of this Article, Fig. 3 was incorrectly duplicated as Fig. 7. Also, the legend to Fig. 7 panel 'e' should read 'Production yield of cis, cis-muconic acid (MA) from glucose (g of produced $\mathrm{MA} \mathrm{g}^{-1}$ of consumed glucose). Red, orange, yellow, purple, and blue symbols indicate the results of cultivation in $0 \%, 25 \%, 50 \%, 75 \%$, and $100 \%$ glucose medium, respectively, in ' $\mathbf{a}-\mathbf{e}$ '; ' $\mathbf{a}-\mathbf{h}$ ' were referred to in the original version. This has been corrected in both the PDF and HTML versions of the Article.

Published online: 20 February 2020

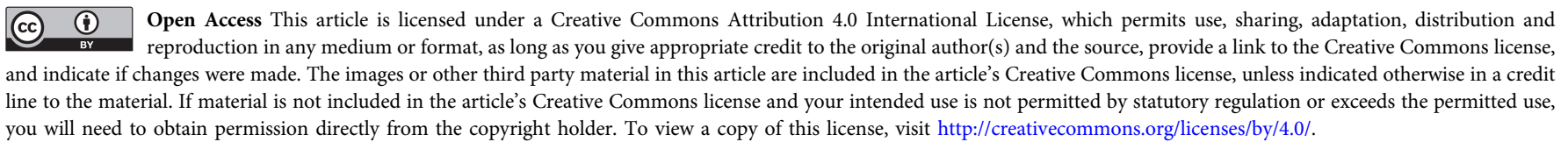

(C) The Author(s) 2020 